\title{
Prevalence of the Metabolic Syndrome among Korean Workers by Occupational Group: Fifth Korean National Health and Nutrition Examination Survey (KNHANES) 2010
}

\author{
Ji Young Ryu', Sukwoo Hong ${ }^{1}$, Chang-Hyo Kim², Sangyoon Lee', Jeong-Ho Kim³ , Jong-Tae Lee
} and Dae Hwan Kim ${ }^{1 *}$

\begin{abstract}
Objectives: The prevalence of the metabolic syndrome has increased rapidly in South Korea over the past 10 years. However, the occurrence of the metabolic syndrome in workers grouped according to the specific type of work is not well understood in Korea. In this study, we assessed the differences in the prevalence of the metabolic syndrome by occupational group and evaluated the risk of the metabolic syndrome among occupational groups.

Methods: From the Fifth Korean National Health and Nutrition Examination Survey (2010), 3,303 employed participants were included in this study. The unadjusted and age-adjusted prevalences of the metabolic syndrome were estimated and multiple logistic regression analysis was conducted using the presence of the metabolic syndrome as a dependent variable, and adjusting for age, education level, household income, drinking behavior, smoking status, physical activity, work hours, and work scheduling pattern.
\end{abstract}

Results: Among male workers, non-manual workers had the greatest age-adjusted prevalence $(26.4 \%, 95 \% \mathrm{Cl}$ : 22.3-30.5\%) among the occupational groups. In a logistic regression analysis, male manual workers had a significantly lower odds ratio for the metabolic syndrome relative to non-manual workers (0.59, 95\% Cl: 0.41-0.85).

Conclusion: Our study demonstrated differences in the prevalence of the metabolic syndrome by occupational group and identified the greatest risk for the metabolic syndrome in male non-manual workers.

Keywords: Metabolic syndrome, Prevalence, Workers, Occupation

\section{Introduction}

The metabolic syndrome is a condition that is defined by the combination of five factors: abdominal obesity, hypertension, hypertriglyceridemia, low HDL-cholesterol level, and hyperglycemia [1]. These components of the metabolic syndrome are major risk factors for cardiovascular disease and impaired glucose metabolism [1-4].

Recently, the prevalence of the metabolic syndrome has increased rapidly in South Korea [5,6]. According to a study using the data from the Korean National Health

\footnotetext{
* Correspondence: kimdh@paik.ac.kr

'Department of Occupational and Environmental Medicine, Inje University Haeundae Paik Hospital, 875 Heaundae-ro, Haeundae-Gu, Busan 612-862, South Korea

Full list of author information is available at the end of the article
}

and Nutrition Examination Survey (KNHANES), the age-adjusted prevalence of the metabolic syndrome in 2007 was $31.3 \%$, while the age-adjusted prevalence of the metabolic syndrome in 1998 had been just 24.9\% [6]. It is thought that the rapid increase in the prevalence of the metabolic syndrome may have arisen from lifestyle changes including a westernized diet and physical inactivity [5-7].

On the other hand, recent studies have shown that the prevalence of the metabolic syndrome varies in different occupational groups [8,9]. Among U.S. workers, the unadjusted and age-adjusted prevalences of the metabolic syndrome were greatest in "transportation and material occupations" and "food preparation and food service workers," respectively [9]. In male Spanish workers, the

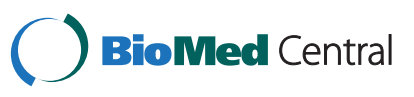


highest age-adjusted prevalence of the metabolic syndrome was found among "machine installers, operators and assemblers" while the prevalence of the metabolic syndrome in female workers was highest among "skilled workers in the agricultural and fishing industries" [8]. It is expected that the prevalence of the metabolic syndrome among workers may differ by country, considering the distinctive working environment of each country. In Korea, only a few studies have assessed the association between the occupational group and the metabolic syndrome. Although previous research by Myoung et al. has evaluated the relationship between occupations and the metabolic syndrome among Korean workers, the study was limited by the fact that the occupations were grouped into manual or non-manual workers only, without considering service and sales workers as a separate work group [10].

In this study, we assessed the differences in the prevalence of the metabolic syndrome and its individual criteria by occupational group and evaluated the risk of the metabolic syndrome among occupational groups using KNHANES data.

\section{Materials and methods Subjects}

Data from the fifth KNHANES, gathered in 2010, was used for the analyses. The KNHANES is a multistage stratified complex design survey of a representative sample of the entire Korean population conducted by the Korea Centers for Disease Control and Prevention. The total number of participants was 8,958 , and the participation rate was $81.9 \%$. Trained interviewers and laboratory technicians conducted surveys in households, including administering questionnaires, performing health examinations, and collecting blood samples. Subjects who were at least 20 years old, who reported having jobs, and who had occupational information were included in this study $(\mathrm{n}=3,667)$. Subjects who reported having no jobs $(n=4,595)$, who had no occupational information $(n=597)$, or who belonged to the armed forces group in the occupational classification $(n=31)$ were excluded from this study. After this exclusion, the workers who were aged below 20 years old were also excluded $(n=68)$. Among 3,667 included workers, 3,303 workers who had fully available data needed for diagnosis of the metabolic syndrome were analyzed. This study was approved by the Institutional Review Board of the Haeundae Paik Hospital.

\section{Occupational classification}

The subjects were classified into nine occupational subgroups based on the KNHANES data. The KNHANES employed the major categories of the Korean Standard Classification of Occupations (KSCO) [11] as the occupational classification. These occupations were grouped into three categories according to their degree of physical work: non-manual workers, service/sales workers, and manual workers. Non-manual workers were managers, professionals and related workers, and clerks. Service workers and sales workers constituted one distinctive group. Manual workers consisted of skilled agricultural, forestry and fishery workers, craft and related trades workers, equipment, machine operating and assembling workers, and elementary workers.

\section{Definition of the metabolic syndrome}

The presence of the metabolic syndrome was determined by the consensus definition [1] incorporating International Diabetes Federation (IDF) worldwide criteria [12] and American Heart Association/National Heart, Lung, and Blood Institute (AHA/NHLBI) criteria [13]. An individual who had any three or more of the following five criteria was defined as having the metabolic syndrome: 1) waist circumference $\geq 90 \mathrm{~cm}$ in men and $\geq 80 \mathrm{~cm}$ in women, 2) blood pressure $\geq 130 / 85 \mathrm{mmHg}$ or receiving anti-hypertensive medication, 3) triglycerides $\geq 150 \mathrm{mg} / \mathrm{dl}$, 4) $\mathrm{HDL}$-cholesterol $<40 \mathrm{mg} / \mathrm{dl}$ in men and $<50 \mathrm{mg} / \mathrm{dl}$ in women, 5) fasting glucose $\geq 100 \mathrm{mg} / \mathrm{dl}$ or taking blood glucose lowering drugs.

\section{Covariates}

Age, level of education, household income, smoking status, high-risk drinking behavior, vigorous physical activity, work hours, work scheduling pattern, and body mass index (BMI) were included as covariates in this study. The levels of education were categorized into elementary school graduates (6-year course), middle school graduates (3-year), high school graduates (3-year), and some university education or above (2 or more years). Household income was classified by quartile as low, low-middle, middle-high, or high levels. Smoking status was divided into non-smokers, ex-smokers, and current smokers. High-risk drinking was defined as at least 7 glasses per day in men and at least 5 glasses per day in women and drinking behavior was categorized into no, once a month, once a week, or every day experience of high-risk drinking. Vigorous physical activity was defined as physical activities over 10 minutes that are more strenuous than usual activities or make one breathless, including carrying heavy loads, running, climbing, fast cycling, fast swimming, soccer, basketball, and so on. This variable was divided into none, below 5 days per week, and at least 5 days per week of vigorous physical activities. Work hours were categorized into 40 hours per week or less and over 40 hours per week. Work schedule patterns were grouped as either daytime fixed work or non-daytime work, including night-time and shift work. BMI was classified into normal/underweight 
(below $25 \mathrm{~kg} / \mathrm{m}^{2}$ ), overweight (between 25 and $30 \mathrm{~kg} / \mathrm{m}^{2}$ ), and obese (more than $30 \mathrm{~kg} / \mathrm{m}^{2}$ ).

\section{Statistical analyses}

The statistical analyses were performed using SAS Enterprise Guide (version 4.2; SAS Institute Inc., Cary, NC) to take into account sample weights and complex sample design effects. All of the data are presented as an estimated percentage (standard error) or estimated mean (standard error) for the demographic variables and each component of the metabolic syndrome. The Rao-Scott chi-squared test was used to compare the prevalence of the categorical variables. The unadjusted and age-adjusted prevalences of the metabolic syndrome for nine occupational groups were estimated. Age-standardization by the direct method was done for the prevalence estimates using 2010 Census population data of Korea. The prevalence estimate for each occupational group was considered significantly higher than that for workers overall if the estimate was above the upper bounds of the $95 \%$ confidence interval for the overall prevalence estimate [14]. For the occupational groups, multiple logistic regression analysis was conducted with the presence of the metabolic syndrome as a dependent variable after stratification for gender. The multiple logistic regression analysis was performed, adjusting for age (continuous variable), level of education, household income, drinking behavior, smoking status, physical activity, work hours, and work schedule pattern.

\section{Results}

Tables 1 and 2 present the demographic characteristics by the major job categories of the KSCO among male and female workers, respectively. In both the male and female workers, "managers", "professional and related" workers, and "clerks" had high levels of education and household income. Among the male workers, the proportion doing vigorous physical activity at least 5 days per week was lowest in the "managers" (3.5\%), followed by "professional and related" workers (3.8\%) and "clerks" $(5.8 \%)$ and highest in the "skilled agricultural, forestry and fishery workers" (17.5\%), followed by "elementary" workers (14.8\%), sales workers (12.7\%), and service workers $(12.3 \%)$. The proportion of those with less than 40 work hours per week was higher in the "managers" (48.4\%), "professional and related" workers (41\%), and "clerks" (42.6\%) than in the other work groups in the men. Service workers reported the highest proportion of non-day work in both the male (51.4\%) and female (29.8\%) workers. "Managers", "professional and related" workers, and "clerks" were more likely to be overweight or obese than those of the other occupational groups in the male workers. On the other hand, in female workers, the proportion of overweight or obese individuals was smaller among the "managers", "professional and related" workers, and "clerks" than the other occupational groups.

The prevalence estimates of the metabolic syndrome by demographic characteristics stratified by gender are shown in Table 3. Among the male workers, the subgroup with the highest prevalence of the metabolic syndrome was the obese group (68.3\%), followed by the overweight group (39.1\%) and the group engaging in high-risk drinking everyday (38.1\%). In the female workers, the group of those aged $\geq 70$ years $(53.5 \%)$ showed the highest prevalence of the metabolic syndrome, followed by the obese group (46.1\%).

Unadjusted and age-adjusted prevalence estimates for each occupational group stratified by gender are presented in Table 4. In the male workers, the overall unadjusted and age-adjusted prevalence estimates of the metabolic syndrome were $24.1 \%$ (95\% CI: $21.7-26.5 \%$ ) and $23.2 \%$ (95\% CI: $21.0-25.5 \%$ ), respectively. The prevalence estimates in male non-manual workers were $26.3 \%$ (95\% CI: $22.3-30.2 \%)$ and $26.4 \%$ (95\% CI: $22.3-30.5 \%)$, respectively, after age adjustment, which were the highest among the three occupational groups. In the occupational subgroups, "equipment, machine operation and assembling" workers showed the highest unadjusted prevalence of the metabolic syndrome $(35.4 \%, 95 \% \mathrm{CI}$ : 28.4-42.4\%), followed by "managers" (31.7\%, 95\% CI: 18.1-45.3\%) and "clerks" (26.9\%, 95\% CI: 21.0-32.8\%). The highest age-adjusted prevalence for male workers was found in "professional and related" workers (27.3\%, 95\% CI: 20.9-33.6\%), followed by "equipment, machine operation and assembling" workers (26.5\%, 95\% CI: 21.1-32.0\%) and "managers" (26.5\%, 95\% CI: 14.2-38.8\%).

In the female workers, the estimates of overall unadjusted and age-adjusted prevalence of the metabolic syndrome were $17.3 \%$ (95\% CI: $14.8-19.8 \%$ ) and 19.5\% (95\% CI: 17.3-21.6\%), respectively. Among the female workers, the age-adjusted prevalence of the metabolic syndrome was similar in both non-manual $(20.8 \%, 95 \%$ CI: $11.3-30.2 \%)$ and manual (20.8\%, 95\% CI: $16.9-24.7 \%)$ workers, while the unadjusted prevalence was highest in manual workers $(28.4 \%, 95 \%$ CI: $23.9-32.9 \%)$ and was lowest in non-manual workers (7.7\%, 95\% CI: 4.4-10.9\%). In the individual occupational groups, the highest unadjusted prevalence was documented in "skilled agricultural, forestry and fishery" workers (39.2\%, 95\% CI: 31.8-46.6\%), followed by "elementary" workers $(25.0 \%, 95 \%$ CI: 19.1$31.0 \%)$ and "craft and related trades" workers (19.1\%, 95\% CI: 4.4-33.8\%). "Professional and related" workers showed the highest age-adjusted prevalence of the metabolic syndrome among the female workers $(27.9 \%, 95 \% \mathrm{CI}$ : 21.5-34.3\%), followed by "skilled agricultural, forestry and fishery” workers (22.0\%, 95\% CI: 15.2-28.9). 
Table 1 Demographic characteristics by major category of the Korean Standard Classification of Occupations among male workers

\begin{tabular}{|c|c|c|c|c|c|c|c|c|c|c|c|}
\hline & Managers & $\begin{array}{l}\text { Professional and } \\
\text { related workers }\end{array}$ & Clerks & $\begin{array}{c}\text { Service } \\
\text { workers }\end{array}$ & $\begin{array}{c}\text { Sales } \\
\text { workers }\end{array}$ & $\begin{array}{l}\text { Skilled agricultural, } \\
\text { forestry and } \\
\text { fishery workers }\end{array}$ & $\begin{array}{l}\text { Craft and related } \\
\text { trades workers }\end{array}$ & $\begin{array}{l}\text { Equipment, machine } \\
\text { operation and } \\
\text { assembling workers }\end{array}$ & $\begin{array}{c}\text { Elementary } \\
\text { workers }\end{array}$ & Total & $\mathrm{p}$-value \\
\hline Age (years)* & $50.9(1.3)$ & $38.2(0.7)$ & $39.1(0.7)$ & $36.8(1.4)$ & $41.2(1.2)$ & $56.4(1.4)$ & $42.7(0.9)$ & $45.1(1.1)$ & $46.1(1.7)$ & $44.1(0.4)$ & $<0.001$ \\
\hline Education & & & & & & & & & & & $<0.001$ \\
\hline$\leq$ Elementary school & $0.0(0.0)$ & $0.6(0.4)$ & $0.4(0.4)$ & $4.9(2.1)$ & $6.5(2.1)$ & $41.5(3.3)$ & $14.2(2.7)$ & $7.7(1.8)$ & $21.7(4.3)$ & $10.7(1.1)$ & \\
\hline Middle school & $16.3(5.6)$ & $0.4(0.3)$ & $1.7(1.0)$ & $6.4(2.4)$ & $7.6(2.2)$ & $20.8(3.9)$ & $13.9(2.5)$ & $19.7(3.0)$ & $17.5(3.6)$ & $10.3(0.9)$ & \\
\hline High school & $31.8(6.7)$ & $21.0(3.6)$ & $27.6(3.4)$ & $56.3(5.4)$ & $45.7(4.4)$ & $24.9(3.2)$ & $53.3(4.0)$ & $56.9(4.0)$ & $50.5(4.9)$ & $39.2(1.5)$ & \\
\hline$\geq$ University & $51.8(6.7)$ & $78.0(3.6)$ & $70.3(3.6)$ & $32.4(4.8)$ & $40.2(3.9)$ & $12.8(2.8)$ & $18.6(3.0)$ & $15.7(3.2)$ & $10.3(2.8)$ & $39.8(1.7)$ & \\
\hline Household income & & & & & & & & & & & $<0.001$ \\
\hline Low & $3.7(2.7)$ & $6.7(1.8)$ & $2.7(1.1)$ & $6.5(2.7)$ & $9.3(2.2)$ & $24.1(4.0)$ & $12.3(2.8)$ & $8.6(2.4)$ & $26.5(4.2)$ & $10.9(1.0)$ & \\
\hline Low-middle & $10.6(4.4)$ & $17.1(2.7)$ & $24.5(2.8)$ & $34.6(2.7)$ & $27.6(3.6)$ & $28.2(4.3)$ & $35.4(3.7)$ & $26.9(3.5)$ & $29.2(4.3)$ & $26.4(1.4)$ & \\
\hline Middle-high & $29.2(7.2)$ & $39.0(3.0)$ & $33.9(3.1)$ & $25.0(4.4)$ & $36.1(4.3)$ & $18.2(2.6)$ & $29.0(3.4)$ & $37.6(3.6)$ & $29.5(4.9)$ & $31.9(1.4)$ & \\
\hline High & $56.5(6.1)$ & $37.3(2.9)$ & $38.9(3.7)$ & $33.9(5.2)$ & $27.0(3.7)$ & $29.5(4.2)$ & $23.4(3.3)$ & $26.9(3.4)$ & $14.8(3.6)$ & $30.7(1.6)$ & \\
\hline High-risk drinking & & & & & & & & & & & 0.002 \\
\hline Never & $17.5(5.2)$ & $12.7(2.1)$ & $14.1(2.3)$ & $7.4(2.6)$ & $13.0(2.7)$ & $18.1(3.6)$ & $14.1(2.5)$ & $14.8(2.3)$ & $26.4(4.6)$ & $14.8(1.0)$ & \\
\hline Once a month & $13.4(6.3)$ & $46.3(3.4)$ & $38.9(3.3)$ & $44.4(5.8)$ & $33.7(4.0)$ & $32.6(4.3)$ & $42.5(4.5)$ & $39.2(4.3)$ & $35.9(5.5)$ & $40.0(1.5)$ & \\
\hline Once a weak & $30.0(6.6)$ & $30.2(3.2)$ & $38.3(3.6)$ & $35.5(5.3)$ & $43.6(4.3)$ & $26.2(4.1)$ & $28.8(3.8)$ & $29.7(3.5)$ & $20.2(4.7)$ & $31.8(1.2)$ & \\
\hline Everyday & $9.1(4.2)$ & $10.7(2.0)$ & $8.7(2.2)$ & $12.7(3.6)$ & $9.8(2.6)$ & $23.2(3.3)$ & $14.6(2.7)$ & $16.3(3.2)$ & $17.5(4.0)$ & $13.4(1.1)$ & \\
\hline Smoking status & & & & & & & & & & & 0.027 \\
\hline Non-smoker & $15.0(4.4)$ & $23.7(2.4)$ & $17.7(2.6)$ & $18.7(3.9)$ & $17.9(3.1)$ & $13.9(2.8)$ & $12.0(2.4)$ & $10.9(2.5)$ & $15.5(3.9)$ & $16.5(1.0)$ & \\
\hline Ex-smoker & $41.1(6.1)$ & $30.1(2.9)$ & $31.6(2.8)$ & $23.9(4.4)$ & $31.8(3.7)$ & $41.8(3.0)$ & $32.7(3.6)$ & $34.4(3.1)$ & $29.5(4.3)$ & $32.6(1.3)$ & \\
\hline Current smoker & $43.9(6.0)$ & $46.3(3.4)$ & $50.8(2.9)$ & $57.4(5.1)$ & $50.3(4.4)$ & $44.3(3.5)$ & $55.4(3.7)$ & $54.7(3.4)$ & $55.0(5.3)$ & $50.9(1.4)$ & \\
\hline Vigorous physical activity & & & & & & & & & & & $<0.001$ \\
\hline None & $66.2(6.4)$ & $53.3(3.8)$ & $44.4(3.6)$ & $52.1(5.1)$ & $50.2(3.5)$ & $60.7(3.3)$ & $53.3(4.2)$ & $49.4(3.7)$ & $58.8(5.2)$ & $52.7(1.4)$ & \\
\hline$<5$ days per week & $30.3(6.1)$ & $42.8(3.9)$ & $49.7(3.6)$ & $35.7(5.2)$ & $37.1(4.1)$ & $21.8(2.8)$ & $36.1(4.1)$ & $41.4(3.7)$ & $26.4(5.2)$ & $37.6(1.4)$ & \\
\hline$\geq 5$ days per week & $3.5(2.1)$ & $3.8(1.2)$ & $5.8(1.4)$ & $12.3(3.6)$ & $12.7(3.0)$ & $17.5(2.6)$ & $10.7(2.2)$ & $9.2(2.4)$ & $14.8(4.1)$ & $9.7(0.8)$ & \\
\hline Work hours & & & & & & & & & & & $<0.001$ \\
\hline$\leq 40$ hours per week & $48.8(6.5)$ & $41.0(3.6)$ & $42.6(3.3)$ & $28.5(4.7)$ & $28.3(3.9)$ & $37.8(4.4)$ & $25.6(2.9)$ & $23.6(3.2)$ & $39.4(4.8)$ & $34.4(1.3)$ & \\
\hline$>40$ hours per week & $51.2(6.5)$ & $59.0(3.6)$ & $57.4(3.3)$ & $71.5(4.7)$ & $71.7(3.9)$ & $62.2(4.4)$ & $74.4(2.9)$ & $76.4(3.2)$ & $60.6(4.8)$ & $65.6(1.3)$ & \\
\hline Work schedule pattern & & & & & & & & & & & $<0.001$ \\
\hline Day & $92.0(4.5)$ & $81.7(2.5)$ & $90.9(2.2)$ & $48.6(5.7)$ & $84.8(3.0)$ & $99.8(0.2)$ & $87.2(3.5)$ & $72.9(3.3)$ & $68.5(4.1)$ & $82.2(1.2)$ & \\
\hline Night/overnight & $3.7(2.0)$ & $13.6(2.3)$ & $2.6(1.1)$ & $26.3(5.3)$ & $14.2(3.0)$ & $0.1(0.1)$ & $2.0(1.3)$ & $8.7(2.0)$ & $11.3(2.8)$ & $8.6(0.8)$ & \\
\hline
\end{tabular}


Table 1 Demographic characteristics by major category of the Korean Standard Classification of Occupations among male workers (Continued)

\begin{tabular}{|c|c|c|c|c|c|c|c|c|c|c|c|}
\hline Shift & 0.0 & $1.5(0.9)$ & $5.2(1.9)$ & $15.5(4.1)$ & $0.3(0.2)$ & 0.0 & $8.4(2.7)$ & $11.3(2.5)$ & $19.4(3.2)$ & $6.3(0.8)$ & \\
\hline Others & $4.2(4.1)$ & $3.2(1.0)$ & $1.3(0.7)$ & $9.6(2.7)$ & $0.7(0.5)$ & $0.1(0.1)$ & $2.4(2.0)$ & $7.1(2.2)$ & $0.8(0.7)$ & $2.9(0.5)$ & \\
\hline BMI category & & & & & & & & & & & 0.230 \\
\hline Normal/underweight & $55.2(7.6)$ & $56.6(3.0)$ & $58.1(3.5)$ & $60.5(5.8)$ & $62.8(4.2)$ & $68.5(3.5)$ & $61.4(3.3)$ & $62.2(3.7)$ & $67.0(4.5)$ & $61.3(1.4)$ & \\
\hline Overweight & $37.5(6.4)$ & $39.3(2.9)$ & $36.4(3.6)$ & $37.7(5.7)$ & $30.5(3.7)$ & $30.8(3.4)$ & $35.2(3.2)$ & $33.5(3.6)$ & $30.1(4.5)$ & $34.7(1.3)$ & \\
\hline Obese & $7.3(3.7)$ & $4.1(1.3)$ & $5.5(1.7)$ & $1.8(1.3)$ & $6.7(2.0)$ & $0.7(0.5)$ & $3.4(1.5)$ & $4.3(1.5)$ & $2.9(1.4)$ & $3.9(0.6)$ & \\
\hline
\end{tabular}

*Estimated mean (standard error) 
Table 2 Demographic characteristics by major category of the Korean Standard Classification of Occupations among female workers

\begin{tabular}{|c|c|c|c|c|c|c|c|c|c|c|c|}
\hline & Managers & $\begin{array}{l}\text { Professional and } \\
\text { related workers }\end{array}$ & Clerks & $\begin{array}{l}\text { Service } \\
\text { workers }\end{array}$ & $\begin{array}{c}\text { Sales } \\
\text { workers }\end{array}$ & $\begin{array}{l}\text { Skilled agricultural, } \\
\text { forestry and } \\
\text { fishery workers }\end{array}$ & $\begin{array}{l}\text { Craft and related } \\
\text { trades workers }\end{array}$ & $\begin{array}{l}\text { Equipment, machine } \\
\text { operation and } \\
\text { assembling workers }\end{array}$ & $\begin{array}{l}\text { Elementary } \\
\text { workers }\end{array}$ & Total & $\mathrm{p}$-value \\
\hline Age $(y r)^{*}$ & $54.1(3.9)$ & $34.2(0.6)$ & $33.7(0.7)$ & $43.2(1.0)$ & $43.2(1.0)$ & $57.4(1.5)$ & $49.1(2.9)$ & $41.8(2.4)$ & $51.7(1.0)$ & $45.4(0.6)$ & $<0.001$ \\
\hline Education & & & & & & & & & & & $<0.001$ \\
\hline$\leq$ Elementary school & $0.0(0.0)$ & $0.6(0.3)$ & $0.3(0.3)$ & $21.7(3.3)$ & $18.4(2.6)$ & $67.2(4.7)$ & $30.4(9.7)$ & $16.5(7.4)$ & $39.8(3.0)$ & $22.3(1.9)$ & \\
\hline Middle school & $18.2(15.1)$ & $0.6(0.4)$ & $2.2(1.0)$ & $18.3(2.9)$ & $13.3(2.5)$ & $15.5(4.5)$ & $34.5(8.4)$ & $17.2(7.6)$ & $18.5(3.4)$ & $11.6(1.1)$ & \\
\hline High school & $18.4(12.9)$ & $17.1(2.5)$ & $50.1(3.9)$ & $47.3(3.6)$ & $47.6(3.7)$ & $15.0(4.2)$ & $31.8(7.6)$ & $60.4(10.3)$ & $34.7(3.7)$ & $34.5(1.7)$ & \\
\hline$\geq$ University & $63.3(18.0)$ & $81.7(2.5)$ & $47.4(4.1)$ & $12.6(2.7)$ & $20.7(3.0)$ & $2.3(1.4)$ & $3.3(2.3)$ & $5.8(4.1)$ & $6.9(1.8)$ & $31.5(1.8)$ & \\
\hline Household income & & & & & & & & & & & $<0.001$ \\
\hline Low & $0.0(0.0)$ & $4.0(1.4)$ & $2.6(1.2)$ & $10.0(2.3)$ & $12.1(2.3)$ & $35.7(4.9)$ & $30.6(9.4)$ & $0.0(0.0)$ & $25.1(2.9)$ & $14.2(1.3)$ & \\
\hline Low-middle & $1.2(1.2)$ & $17.2(2.7)$ & $20.7(3.8)$ & $31.1(3.6)$ & $23.0(3.8)$ & $25.6(3.2)$ & $23.6(6.1)$ & $23.9(7.7)$ & $36.9(3.4)$ & $25.4(1.5)$ & \\
\hline Middle-high & $14.6(13.3)$ & $37.4(3.3)$ & $36.8(4.2)$ & 30.7 (3.8) & $35.4(3.8)$ & $18.0(3.3)$ & $28.2(7.6)$ & $45.2(10.9)$ & $22.6(2.5)$ & $30.8(1.5)$ & \\
\hline High & $84.2(13.4)$ & $41.4(3.4)$ & $40.0(3.8)$ & $28.2(3.3)$ & $29.6(3.7)$ & $20.7(3.2)$ & $17.6(6.5)$ & $30.9(9.5)$ & $15.4(2.6)$ & $29.6(1.6)$ & \\
\hline High-risk drinking & & & & & & & & & & & $<0.001$ \\
\hline Never & 77.4 (15.6) & $39.6(3.5)$ & $31.7(3.9)$ & 35.9 (3.9) & $43.6(3.8)$ & $57.0(5.4)$ & $18.5(6.7)$ & $37.1(11.3)$ & $42.7(5.0)$ & $39.8(1.7)$ & \\
\hline Once a month & $22.6(15.6)$ & $45.0(3.7)$ & $52.9(4.3)$ & $43.5(4.0)$ & $37.8(4.2)$ & $38.4(4.4)$ & $58.6(9.3)$ & $59.8(11.7)$ & $35.9(3.9)$ & $43.6(4.6)$ & \\
\hline Once a weak & $0.0(0.0)$ & $15.4(3.2)$ & $14.7(3.0)$ & $13.6(2.6)$ & $17.2(3.3)$ & $4.0(2.2)$ & $19.5(8.5)$ & $3.1(3.1)$ & $14.3(3.3)$ & $13.8(1.3)$ & \\
\hline Everyday & $0.0(0.0)$ & $0.0(0.0)$ & $0.7(0.7)$ & $7.0(2.0)$ & $1.3(1.3)$ & $0.6(0.6)$ & $3.4(2.6)$ & $0.0(0.0)$ & $7.1(3.0)$ & $2.7(0.6)$ & \\
\hline Smoking status & & & & & & & & & & & 0.015 \\
\hline Non-smoker & $100.0(0.0)$ & $88.5(1.9)$ & $86.3(2.7)$ & $86.0(3.1)$ & $83.4(3.0)$ & $96.4(1.7)$ & $91.8(5.6)$ & 96.7 (3.3) & $83.0(3.2)$ & $87.4(1.2)$ & \\
\hline Ex-smoker & $0.0(0.0)$ & $7.5(1.6)$ & $7.9(2.2)$ & $4.9(1.7)$ & $9.5(2.4)$ & $2.3(1.4)$ & $8.0(5.6)$ & $0.0(0.0)$ & $5.4(1.7)$ & $6.3(0.8)$ & \\
\hline Current smoker & $0.0(0.0)$ & $4.0(1.1)$ & $5.8(1.8)$ & $9.2(2.9)$ & $7.1(2.2)$ & $1.3(0.8)$ & $0.1(0.1)$ & $3.3(3.3)$ & $11.6(3.1)$ & $6.3(0.9)$ & \\
\hline Vigorous physical activity & & & & & & & & & & & 0.007 \\
\hline None & $55.8(18.7)$ & $74.1(3.1)$ & $70.2(3.7)$ & $70.1(3.7)$ & $69.5(3.8)$ & $76.1(4.1)$ & $86.3(5.4)$ & $61.5(8.8)$ & $75.0(3.2)$ & $72.9(1.4)$ & \\
\hline$<5$ days per week & $44.2(18.7)$ & $21.8(2.9)$ & $26.2(3.6)$ & $20.4(3.6)$ & $23.7(3.3)$ & $11.4(2.7)$ & $6.5(3.6)$ & $23.9(8.4)$ & $16.2(2.4)$ & $19.8(1.2)$ & \\
\hline$\geq 5$ days per week & $0.0(0.0)$ & $4.1(1.3)$ & $3.6(1.4)$ & $9.5(2.3)$ & $6.8(2.0)$ & $12.5(3.3)$ & $7.2(4.3)$ & $14.6(5.6)$ & $8.8(2.0)$ & $7.3(0.8)$ & \\
\hline Work hours & & & & & & & & & & & $<0.001$ \\
\hline$\leq 40$ hours per week & $80.4(13.9)$ & $57.0(3.5)$ & $52.4(4.2)$ & $33.7(4.0)$ & $48.9(4.0)$ & $50.9(4.0)$ & $29.4(7.3)$ & $35.9(9.9)$ & $64.5(3.6)$ & $51.2(1.6)$ & \\
\hline$>40$ hours per week & $19.6(13.9)$ & $43.0(3.5)$ & $47.6(4.2)$ & $66.3(4.0)$ & $51.1(4.0)$ & $49.1(4.0)$ & $70.6(7.3)$ & $64.1(9.9)$ & $35.5(3.6)$ & $48.8(1.6)$ & \\
\hline Work schedule pattern & & & & & & & & & & & $<0.001$ \\
\hline Day & $93.8(5.4)$ & $74.8(3.2)$ & $94.3(2.0)$ & $70.2(3.7)$ & $77.7(3.4)$ & $99.0(0.7)$ & $93.0(4.6)$ & $74.2(9.6)$ & $85.8(2.7)$ & $82.7(1.5)$ & \\
\hline Night/overnight & $5.1(5.1)$ & $18.9(3.0)$ & $3.2(1.5)$ & $24.5(3.4)$ & $15.6(2.9)$ & 0.0 & $4.6(2.6)$ & $6.1(3.8)$ & $12.0(2.6)$ & $12.9(1.3)$ & \\
\hline
\end{tabular}


Table 2 Demographic characteristics by major category of the Korean Standard Classification of Occupations among female workers (Continued)

\begin{tabular}{|c|c|c|c|c|c|c|c|c|c|c|c|}
\hline Shift & $1.1(1.2)$ & $3.8(1.5)$ & $1.7(1.3)$ & $2.2(0.9)$ & $3.7(1.7)$ & $0.3(0.3)$ & $2.4(2.4)$ & $8.1(6.1)$ & $1.2(0.6)$ & $2.4(0.5)$ & \\
\hline Others & 0.0 & $2.5(1.0)$ & $0.7(0.5)$ & $3.1(1.2)$ & $3.0(1.4)$ & $0.7(0.6)$ & 0.0 & $11.6(9.0)$ & $1.0(0.5)$ & $2.0(0.4)$ & \\
\hline BMI category & & & & & & & & & & & 0.009 \\
\hline Normal/underweight & $82.8(11.7)$ & $82.9(2.5)$ & $79.7(3.2)$ & $72.2(3.2)$ & $68.5(4.0)$ & $66.8(4.4)$ & $69.4(8.0)$ & $70.3(9.6)$ & $70.9(2.6)$ & $74.2(1.3)$ & \\
\hline Overweight & $17.2(11.7)$ & $14.5(2.2)$ & $15.0(2.6)$ & $22.5(3.0)$ & $27.6(4.0)$ & $26.4(3.3)$ & $30.5(8.0)$ & $17.3(7.8)$ & $24.4(2.7)$ & $21.2(1.1)$ & \\
\hline Obese & $0.0(0.0)$ & $2.6(1.2)$ & $5.2(1.9)$ & $5.3(1.8)$ & $3.9(1.5)$ & $6.8(1.9)$ & $0.1(0.1)$ & $12.4(7.0)$ & $4.7(1.3)$ & $4.5(0.7)$ & \\
\hline
\end{tabular}

*Estimated mean (standard error). 
Table 3 The prevalence of the metabolic syndrome according to demographic characteristics

\begin{tabular}{|c|c|c|c|c|}
\hline \multirow[b]{2}{*}{ Demographics } & \multicolumn{2}{|l|}{ Male } & \multicolumn{2}{|l|}{ Female } \\
\hline & Number ${ }^{*}+$ & 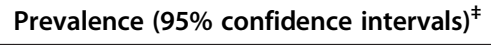 & Number $^{* \dagger}$ & Prevalence ( $95 \%$ confidence intervals) \\
\hline \multicolumn{5}{|l|}{ Age group } \\
\hline 20-29 years & $152 / 1,942,533$ & $9.2(4.1-14.3)$ & $231 / 1,733,502$ & $3.5(0.7-6.2)$ \\
\hline 30-39 years & $428 / 3,276,013$ & $17.8(13.5-22.1)$ & $291 / 1,634,571$ & $5.5(2.0-9.0)$ \\
\hline 40-49 years & $477 / 3,659,709$ & $25.9(21.3-30.4)$ & $371 / 2,433,142$ & $12.5(8.2-16.8)$ \\
\hline $50-59$ years & $392 / 2,681,787$ & $35.7(30.2-41.2)$ & $374 / 1,891,022$ & $30.8(25.2-36.5)$ \\
\hline $60-69$ years & $297 / 1,176,249$ & $36.4(29.5-43.3)$ & $188 / 758,966$ & $38.0(30.1-45.8)$ \\
\hline$\geq 70$ years & $113 / 415,885$ & $17.4(9.1-25.8)$ & $93 / 373,407$ & $53.5(43.5-63.4)$ \\
\hline \multicolumn{5}{|l|}{ Education } \\
\hline$\leq$ Elementary school & $262 / 1,377,314$ & $28.7(21.5-35.8)$ & $407 / 1,912,522$ & $40.9(35.6-46.2)$ \\
\hline Middle school & 218/1,385,292 & $35.9(29.0-42.8)$ & $175 / 989,224$ & $24.4(16.6-32.3)$ \\
\hline High school & $637 / 5,078,713$ & $20.1(16.4-23.7)$ & $491 / 3,089796$ & $9.3(6.1-12.5)$ \\
\hline$\geq$ University & $742 / 5,310,859$ & $23.6(19.9-27.4)$ & $481 / 2,833,069$ & $7.6(4.2-11.1)$ \\
\hline \multicolumn{5}{|l|}{ Household income } \\
\hline Low & 224/1,362,396 & $25.9(18.9-32.9)$ & $239 / 1,171,008$ & $35.3(28.6-41.9)$ \\
\hline Low-middle & $476 / 3,488,782$ & $21.4(16.9-25.8)$ & $366 / 2,177,822$ & $19.8(15.3-24.2)$ \\
\hline Middle-high & $568 / 4,173,072$ & $23.8(19.4-28.2)$ & $448 / 2,712,764$ & $14.3(10.7-18.0)$ \\
\hline High & $575 / 4,051,739$ & $25.5(21.1-29.9)$ & $481 / 2,648,160$ & $10.4(7.1-13.7)$ \\
\hline \multicolumn{5}{|l|}{ High-risk drinking } \\
\hline Never & $275 / 1,681,687$ & $14.7(9.6-19.9)$ & $473 / 2,633,998$ & $15.4(11.2-19.7)$ \\
\hline Once a month & $644 / 4,713,082$ & $21.2(17.4-24.9)$ & $494 / 2,956,125$ & $12.8(9.3-16.4)$ \\
\hline Once a weak & $490 / 3,727,662$ & $26.0(21.6-30.4)$ & $130 / 932,867$ & $17.4(9.6-25.2)$ \\
\hline Everyday & $226 / 1,602,909$ & $38.1(30.7-45.5)$ & $23 / 152,018$ & $31.1(8.8-53.4)$ \\
\hline \multicolumn{5}{|l|}{ Smoking status } \\
\hline Non-smoker & $320 / 2,194,133$ & $19.5(14.4-24.5)$ & $1,394 / 7,721,944$ & $17.5(14.9-20.2)$ \\
\hline Ex-smoker & $697 / 4,369,598$ & $27.0(22.9-31.0)$ & $81 / 546,406$ & $16.0(7.0-25.0)$ \\
\hline Current smoker & $841 / 6,585,245$ & $23.7(20.1-27.3)$ & $78 / 547,078$ & $15.8(6.7-24.9)$ \\
\hline \multicolumn{5}{|l|}{ Vigorous physical activity } \\
\hline None & $1,035 / 6,872,680$ & $25.0(21.9-28.1)$ & $1,144 / 6,444,936$ & $17.8(14.4-20.7)$ \\
\hline$<5$ days per week & $659 / 4,976,027$ & $23.7(19.5-28.0)$ & $293 / 1,726,743$ & $17.3(12.5-22.2)$ \\
\hline$\geq 5$ days per week & $164 / 1,300,269$ & $20.2(12.7-27.8)$ & $115 / 633,191$ & $14.8(7.9-21.7)$ \\
\hline \multicolumn{5}{|l|}{ Work hours } \\
\hline$\leq 40$ hours per week & $666 / 4,555,070$ & $22.5(18.6-26.5)$ & $819 / 4,514,730$ & $17.3(13.9-20.7)$ \\
\hline$>40$ hours per week & 1,193/8,597,108 & $24.9(21.8-28.0)$ & $735 / 4,309,882$ & $17.3(14.3-20.4)$ \\
\hline \multicolumn{5}{|l|}{ Work schedule pattern } \\
\hline Day working & $1,526 / 10,805,797$ & $24.5(21.9-271)$ & $1,312 / 7,305,852$ & $18.3(15.4-21.2)$ \\
\hline Non-day working & $328 / 2,319,154$ & $22.0(16.6-27.4)$ & $238 / 1,501,207$ & $12.4(6.8-18.1)$ \\
\hline \multicolumn{5}{|l|}{ BMI category } \\
\hline Underweight & $40 / 272,239$ & $0.0(0.0-0.0)$ & $85 / 539,963$ & $0.0(0.0-0.0)$ \\
\hline Normal & $1,099 / 7,673,502$ & $12.9(10.3-15.4)$ & $1,053 / 6,039,293$ & $9.4(7.5-11.4)$ \\
\hline Overweight & $654 / 4,672,170$ & $39.1(34.3-43.9)$ & $350 / 1,858,859$ & $41.9(35.7-48.1)$ \\
\hline Obese & $61 / 500,005$ & $68.3(54.1-82.5)$ & $65 / 384,483$ & $46.1(32.3-59.9)$ \\
\hline
\end{tabular}

"Subject number/estimated population number. ${ }^{\dagger}$ Subject number varies due to item which was not answered. ${ }^{*}$ Unadjusted prevalence of the metabolic syndrome (95\% confidence intervals). 
Table 4 Unadjusted and age-adjusted prevalence of the metabolic syndrome by category of the Korean Standard Classification of Occupations stratified by gender

\begin{tabular}{|c|c|c|c|}
\hline \multirow[b]{2}{*}{ Occupational groups } & \multirow[b]{2}{*}{ Number ${ }^{*}$} & \multicolumn{2}{|c|}{ Prevalence of the metabolic syndrome ( $95 \%$ confidence intervals) } \\
\hline & & Unadjusted & Age-adjusted \\
\hline Overall male workers & $1,859 / 13,152,179$ & $24.1(21.7-26.5)$ & $23.2(21.0-25.5)$ \\
\hline Non-manual workers & $676 / 4,905,005$ & $26.3(22.3-30.2)^{\dagger}$ & $26.4(22.3-30.5)^{\dagger}$ \\
\hline Managers & $61 / 362,106$ & $31.7(18.1-45.3)^{\dagger}$ & $26.5(14.2-38.8)^{\dagger}$ \\
\hline Professional and related workers & $335 / 2,531,917$ & $25.0(19.3-30.8)^{\dagger}$ & $27.3(20.9-33.6)^{+}$ \\
\hline Clerks & $280 / 2,010,981$ & $26.9(21.0-32.8)^{\dagger}$ & $24.6(18.1-31.1)$ \\
\hline Service/sales workers & $304 / 2,302,276$ & $19.9(14.6-25.2)$ & $21.5(15.9-27.1)$ \\
\hline Service workers & $104 / 910,156$ & $15.7(8.1-23.3)$ & $17.5(9.0-26.0)$ \\
\hline Sales workers & $200 / 1,392,120$ & $22.7(15.3-30.0)$ & $23.0(16.2-29.8)$ \\
\hline Manual workers & $879 / 5,944,897$ & $23.9(20.6-27.1)$ & $20.1(17.3-23.0)$ \\
\hline Skilled agricultural, forestry and fishery workers & $272 / 1,589,656$ & $24.3(18.2-30.4)$ & $15.6(10.9-20.2)$ \\
\hline Craft and related trades workers & $206 / 1,733,288$ & $15.7(10.6-20.7)$ & $16.4(11.0-21.9)$ \\
\hline Equipment, machine operating and assembling workers & $240 / 1,597,780$ & $35.4(28.4-42.4)^{\dagger}$ & $26.5(21.1-32.0)^{+}$ \\
\hline Elementary workers & $161 / 1,024,171$ & $19.2(11.3-27.2)$ & $16.9(9.4-24.4)$ \\
\hline Overall female workers & $1,544 / 8,824,612$ & $17.3(14.8-19.8)$ & $19.5(17.3-21.6)$ \\
\hline Non-manual workers & $545 / 3,325,220$ & $7.7(4.4-10.9)$ & $20.8(11.3-30.2)^{\dagger}$ \\
\hline Managers & $8 / 22,859$ & $12.8(-9.1-34.8)$ & $10.6(-6.7-27.9)$ \\
\hline Professional and related workers & $334 / 1,998,628$ & $7.3(3.5-11.2)$ & $27.9(21.5-34.3)^{\dagger}$ \\
\hline Clerks & $203 / 1,303,732$ & $8.1(2.6-13.5)$ & $9.8(2.7-16.9)$ \\
\hline Service/sales workers & $428 / 2,502,853$ & $16.9(12.8-20.9)$ & $18.4(13-22.9)$ \\
\hline Service workers & $215 / 1,340,314$ & $16.9(11.4-22.3)$ & $20.5(14.5-26.6)^{\dagger}$ \\
\hline Sales workers & $213 / 1,162,539$ & $16.9(11.2-22.5)$ & $17.6(11.9-23.2)$ \\
\hline Manual workers & $581 / 2,996,538$ & $28.4(23.9-32.9)^{\dagger}$ & $20.8(16.9-24.7)^{\dagger}$ \\
\hline Skilled agricultural, forestry and fishery workers & $225 / 1,011,474$ & $39.2(31.8-46.6)^{\dagger}$ & $22.0(15.2-28.9)^{+}$ \\
\hline Craft and related trades workers & $42 / 258,235$ & $19.1(4.4-33.8)$ & $12.0(2.8-21.2)$ \\
\hline Equipment, machine operating and assembling workers & $35 / 158,745$ & $7.8(-2.5-18.1)$ & $6.2(-1.7-14.2)$ \\
\hline Elementary workers & $279 / 1,568,083$ & $25.0(19.1-31.0)^{\dagger}$ & $20.0(14.5-25.5)^{+}$ \\
\hline
\end{tabular}

"Subject number/estimated population number. ${ }^{\dagger}$ The prevalence estimate for each occupational group was above the upper bound of the $95 \%$ confidence interval for the overall prevalence estimate.

Table 5 shows the age-adjusted prevalence of the individual components of the metabolic syndrome by occupational group. Among the three occupational groups of non-manual, service/sales, and manual workers, the non-manual workers had the highest prevalence of high blood pressure (40.1\%), hypertriglyceridemia (39.4\%), low HDL-cholesterol level (20.5\%), and hyperglycemia (31.8\%) among the male workers. The highest prevalence of abdominal obesity was found in the service/sales workers among the male workers (28.6\%). In the occupational subgroups, the prevalence estimate of abdominal obesity for male workers was highest in the "managers" (35.4\%), followed by "craft and related trades" workers (31.1\%) and "equipment, machine operation and assembling" workers (31.1\%). "Clerks" also showed the highest prevalence of high blood pressure (51.6\%) and hyperglycemia (34.5\%) for male workers. Among the male workers, the highest prevalence of hypertriglyceridemia was found in the "equipment, machine operation and assembling" workers (44.9\%), and the highest prevalence of low HDLcholesterol levels was identified in the "professional and related" workers $(22.0 \%)$, followed by "equipment, machine operation and assembling" workers (19.5\%). Unlike the male workers, the prevalence of abdominal obesity among the female workers was highest in the "skilled agricultural, forestry and fishery" workers (50.5\%), followed by "service" workers (42.0\%), "professional and related" workers (41.8\%), and "elementary" workers (40.9\%). The highest prevalence of high blood pressure among the female workers was documented in the "craft and related trades" workers (32.3\%). The highest prevalence of hypertriglyceridemia was found in the "managers" (31.8\%). In the female workers, the "skilled agricultural, forestry and fishery" workers (43.0\%) and "professional and 
Table 5 Age-adjusted prevalence of the components of the metabolic syndrome by category of the Korean Standard Classification of Occupations

\begin{tabular}{|c|c|c|c|c|c|c|c|c|c|c|c|}
\hline \multirow[b]{2}{*}{ Components } & \multicolumn{3}{|c|}{ Non-manual workers } & \multicolumn{2}{|c|}{$\begin{array}{c}\text { Service/sales } \\
\text { workers }\end{array}$} & \multicolumn{4}{|c|}{ Manual workers } & \multirow[b]{2}{*}{ Total } & \multirow[b]{2}{*}{ p-value } \\
\hline & Managers & $\begin{array}{l}\text { Professional and } \\
\text { related workers }\end{array}$ & Clerks & $\begin{array}{l}\text { Service } \\
\text { workers }\end{array}$ & $\begin{array}{c}\text { Sales } \\
\text { workers }\end{array}$ & $\begin{array}{l}\text { Skilled agricultural, } \\
\text { forestry and } \\
\text { fishery workers }\end{array}$ & $\begin{array}{l}\text { Craft and related } \\
\text { trades workers }\end{array}$ & $\begin{array}{l}\text { Equipment, machine } \\
\text { operation and } \\
\text { assembling workers }\end{array}$ & $\begin{array}{l}\text { Elementary } \\
\text { workers }\end{array}$ & & \\
\hline \multicolumn{12}{|l|}{ Male workers } \\
\hline \multirow[t]{2}{*}{$W C \geq 90 \mathrm{~cm}(\mathrm{M}) / 80 \mathrm{~cm}(\mathrm{~W})$} & & $24.5(2.5)$ & \multicolumn{3}{|c|}{$28.6(3.5)$} & \multicolumn{4}{|c|}{$24.0(1.9)$} & \multirow{2}{*}{$25.1(1.3)$} & 0.946 \\
\hline & $35.4(8.7)$ & $21.1(2.8)$ & $25.7(3.7)$ & $25.7(4.7)$ & $28.2(3.9)$ & $22.8(3.5)$ & $31.1(4.3)$ & $31.0(3.2)$ & $18.2(4.2)$ & & 0.705 \\
\hline \multirow[t]{2}{*}{$\mathrm{BP} \geq 130 / 85 \mathrm{mmHg}$ or treated } & & $40.1(2.5)$ & & \multicolumn{2}{|c|}{$33.3(3.3)$} & \multicolumn{4}{|c|}{$32.7(1.7)$} & \multirow{2}{*}{$36.3(1.4)$} & 0.036 \\
\hline & $46.0(6.9)$ & $33.2(3.5)$ & $51.6(2.6)$ & $29.8(4.4)$ & $35.5(3.8)$ & $26.6(3.3)$ & $29.0(3.5)$ & $32.7(2.6)$ & $36.2(4.1)$ & & $<0.001$ \\
\hline \multirow[t]{2}{*}{$\mathrm{TG} \geq 150 \mathrm{mg} / \mathrm{dl}$} & & $39.4(2.6)$ & & \multicolumn{2}{|c|}{$35.5(2.7)$} & \multicolumn{4}{|c|}{$35.3(2.2)$} & \multirow{2}{*}{$37.4(1.4)$} & 0.225 \\
\hline & $41.8(8.0)$ & $43.9(3.3)$ & $33.7(3.8)$ & $35.2(5.0)$ & $37.7(3.3)$ & $25.9(4.6)$ & $34.7(4.0)$ & $44.9(2.9)$ & $29.9(4.9)$ & & 0.119 \\
\hline \multirow[t]{2}{*}{$\mathrm{HDL}-\mathrm{C}<40 \mathrm{mg} / \mathrm{dl}$} & & $20.5(2.2)$ & & \multicolumn{2}{|c|}{$13.3(2.5)$} & \multicolumn{4}{|c|}{$18.3(1.8)$} & \multirow{2}{*}{$19.2(1.2)$} & 0.024 \\
\hline & $14.9(4.1)$ & $22.0(3.2)$ & $16.9(2.5)$ & $12.6(3.8)$ & $13.5(3.0)$ & $9.0(1.5)$ & $17.1(3.0)$ & $19.5(2.9)$ & $18.8(4.6)$ & & 0.119 \\
\hline \multirow[t]{2}{*}{$\mathrm{FPG} \geq 100 \mathrm{mg} / \mathrm{dl}$ or treated } & & $31.8(2.5)$ & & \multicolumn{2}{|c|}{$29.3(3.4)$} & \multicolumn{4}{|c|}{$26.2(1.7)$} & \multirow{2}{*}{$28.6(1.3)$} & 0.191 \\
\hline & $31.2(7.6)$ & $31.8(3.2)$ & $34.5(4.1)$ & $26.2(5.1)$ & $29.2(3.8)$ & $20.3(2.4)$ & $25.8(3.5)$ & $27.1(2.8)$ & $26.8(3.4)$ & & 0.284 \\
\hline \multicolumn{12}{|l|}{ Female workers } \\
\hline \multirow[t]{2}{*}{$W C \geq 90 \mathrm{~cm}(\mathrm{M}) / 80 \mathrm{~cm}(\mathrm{~W})$} & & $34.6(4.8)$ & & \multicolumn{2}{|c|}{$39.0(2.5)$} & \multicolumn{4}{|c|}{$41.8(3.3)$} & $260(1)$ & $<0.001$ \\
\hline & $19.5(12.2)$ & $41.8(3.4)$ & $24.6(4.3)$ & $42.0(3.4)$ & $36.9(3.2)$ & $50.5(6.7)$ & $32.0(5.1)$ & $22.4(5.4)$ & $40.9(5.0)$ & $50.0(1.4)$ & $<0.001$ \\
\hline $\mathrm{BP} \geq 130 / 85 \mathrm{mmHg}$ or treated & & $21.0(1.8)$ & & 23.8 & (2.2) & & & $6(1.9)$ & & $256(12)$ & $<0.001$ \\
\hline & $29.4(10.8)$ & $19.0(2.2)$ & $23.2(3.3)$ & $26.4(2.9)$ & $21.3(3.0)$ & $29.0(4.2)$ & $32.3(4.8)$ & $7.1(4.0)$ & $27.0(2.5)$ & $25.0(1.2)$ & $<0.001$ \\
\hline $\mathrm{TG} \geq 150 \mathrm{mg} / \mathrm{dl}$ & & $15.7(2.7)$ & & 23.3 & (2.9) & & & $6(2.1)$ & & & $<0.001$ \\
\hline & $31.8(13.2)$ & $18.4(2.1)$ & $5.6(2.0)$ & $22.7(4.5)$ & $23.6(3.4)$ & $16.1(2.6)$ & $9.5(4.0)$ & $8.3(4.8)$ & $18.4(2.8)$ & $7.9(1.1)$ & $<0.001$ \\
\hline $\mathrm{HDL}-\mathrm{C}<50 \mathrm{mg} / \mathrm{dl}$ & & $34.9(4.7)$ & & 32.2 & (2.9) & & & $.7(3.1)$ & & 1) & $<0.001$ \\
\hline & $15.1(10.8)$ & $43.0(2.6)$ & $21.2(4.3)$ & $33.2(3.6)$ & $33.0(4.0)$ & $43.0(5.8)$ & $37.9(8.4)$ & $16.8(5.1)$ & $32.1(5.1)$ & $32.0(1.4)$ & $<0.001$ \\
\hline $\mathrm{FPG} \geq 100 \mathrm{mg} / \mathrm{dl}$ or treated & & $19.8(4.5)$ & & 16.1 & (1.9) & & & $.0(2.0)$ & & $81(11)$ & $<0.001$ \\
\hline & $9.4(8.6)$ & $24.7(3.7)$ & $15.6(3.5)$ & $15.3(3.1)$ & $17.1(2.8)$ & $14.9(1.8)$ & $9.0(2.2)$ & $15.2(5.8)$ & $20.7(2.5)$ & $10.1(1.1)$ & $<0.001$ \\
\hline
\end{tabular}

Abbreviation: All values were the estimated percentage (standard error). WC waist circumference, $B P$ blood pressure, $T G$ triglyceride, $H D L-C$ high-density lipoprotein cholesterol, $F P G$ fasting plasma glucose, $M$ male, $F$ female. 
related" workers (43.0\%) showed the highest prevalence of low HDL-cholesterol levels. However, the prevalence of low HDL-cholesterol levels among male workers was lowest in the "skilled agricultural, forestry and fishery" workers (9.0\%). The prevalence of hyperglycemia among the female workers was highest in the "professional and related" workers (24.7\%).

Table 6 shows the results of multiple logistic regression analyses. Manual workers were significantly less likely to have the metabolic syndrome (odds ratio: 0.62, 95\% CI: 1.19-5.86) relative to non-manual workers among male workers after adjustment for age, level of education, household income, high risk drinking, smoking status, and vigorous physical activity (Model I). After additional adjustment for work hours and work schedule pattern, both manual (0.63, 95\% CI: 0.39-0.99) and service/sales $(0.59,95 \%$ CI: $0.41-0.85)$ workers had significantly lower odds of having the metabolic syndrome compared to non-manual workers among the male workers (Model II). There were no significant associations between the metabolic syndrome and the occupational group among female workers in either model. When comparing the subgroups of service/sales and manual workers with the non-manual workers as a reference, "service" workers, "craft and related trades" workers, and "elementary" workers showed significantly lower odds among the male workers.

\section{Discussion}

In this study, we assessed the prevalence of the metabolic syndrome by occupational group among Korean workers and evaluated the risk of the metabolic syndrome among each of the occupational groups. Our study demonstrates variability in the prevalence of the metabolic syndrome by occupational group, and found the greatest risk for the metabolic syndrome in male non-manual workers.

Several studies have identified differences in the prevalence of the metabolic syndrome by occupational group [8-10]. In Spanish workers, the presence of the metabolic syndrome after age adjustment was greatest in the "machine installers, operators, and assemblers" (15.1\%)

Table 6 Multiple logistic regression to assess the relationship between occupation and meeting criteria for the metabolic syndrome among Korean workers aged $\geq 20$ years

\begin{tabular}{|c|c|c|}
\hline & \multicolumn{2}{|c|}{ Odds ratio ( $95 \%$ confidence intervals) } \\
\hline & Male & Female \\
\hline \multicolumn{3}{|l|}{ Model I* } \\
\hline \multicolumn{3}{|l|}{ Occupational group } \\
\hline Non-manual workers & 1.00 (reference) & 1.00 (reference) \\
\hline Service/sales workers & $0.66(0.42-1.02)$ & $0.98(0.45-2.12)$ \\
\hline Service workers & $0.49(0.25-0.96)$ & $0.92(0.36-2.32)$ \\
\hline Sales workers & $0.74(0.43-1.28)$ & $1.12(0.51-2.46)$ \\
\hline Manual workers & $0.62(0.44-0.88)$ & $1.00(0.46-2.16)$ \\
\hline Skilled agricultural, forestry and fishery workers & $0.38(0.20-0.70)$ & $1.56(0.56-4.33)$ \\
\hline Craft and related trades workers & $0.39(0.23-0.66)$ & $0.78(0.21-2.83)$ \\
\hline Equipment, machine operating and assembling workers & $1.22(0.82-1.83)$ & $0.88(0.16-4.67)$ \\
\hline Elementary workers & $0.43(0.21-0.91)$ & $0.84(0.38-1.86)$ \\
\hline \multicolumn{3}{|l|}{ Model $I^{\dagger}$} \\
\hline \multicolumn{3}{|l|}{ Occupational groups } \\
\hline Non-manual workers & 1.00 (reference) & 1.00 (reference) \\
\hline Service/sales workers & $0.63(0.39-0.99)$ & $1.04(0.48-2.25)$ \\
\hline Service workers & $0.45(0.22-0.93)$ & $0.98(0.39-2.47)$ \\
\hline Sales workers & $0.73(0.42-1.26)$ & $1.16(0.53-2.57)$ \\
\hline Manual workers & $0.59(0.41-0.85)$ & $1.02(0.47-2.21)$ \\
\hline Skilled agricultural, forestry and fishery workers & $0.37(0.20-0.69)$ & $1.54(0.56-4.25)$ \\
\hline Craft and related trades workers & $0.37(0.22-0.63)$ & $0.78(0.22-2.81)$ \\
\hline Equipment, machine operating and assembling workers & $1.16(0.77-1.75)$ & $0.90(0.17-4.87)$ \\
\hline Elementary workers & $0.42(0.19-0.91)$ & $0.87(0.39-1.94)$ \\
\hline
\end{tabular}

* Model I: Adjusted for age, level of education, household income, high-risk drinking, smoking status, and vigorous physical activity.

${ }^{\dagger}$ Model II: Adjusted for Model I factors, plus work hours, and work schedule. 
group among males, with an overall prevalence of 9.5\% (11.6\% in male workers and $4.1 \%$ in female workers). Manual workers had a higher prevalence than nonmanual workers in both males and females [8]. According to a study of the U.S. working population, the overall prevalence of the metabolic syndrome was $20.6 \%$ (20.2\% in male workers and $21.4 \%$ in female workers) with the greatest unadjusted prevalence among "transportation and material occupations" (33.1\%) and the greatest age-adjusted prevalence among "food preparation and food service workers" (31.1\%) [9]. In a study using the KNHANES (2005) data, the prevalence of the metabolic syndrome among Korean workers was found to be $21.8 \%$ (22.5\% in male worker and $15.9 \%$ in female), and the prevalence was higher in manual workers than in non-manual workers [10].

In this study, non-manual workers showed higher unadjusted and age-adjusted prevalences of the metabolic syndrome than service/sales and manual workers among the male workers. Most of the workers in the nonmanual groups spend most of their work hours sedentary [15]. Furthermore, they may also engage in less physical activity relative to other workers. Actually, the proportion of those engaging in vigorous physical activity at least 5 days a week in these groups was lower than in the other workers in this study.

In the male non-manual group, the "managers" showed the highest prevalence estimate (31.7\%), but the prevalence in the "equipment, machine operating and assembling" workers was the highest (35.4\%) among the nine occupational subgroups before age adjustment. On the other hand, after age adjustment, the highest prevalence was observed in "professional and related" workers (27.3\%), followed by "managers" (26.5\%) and "equipment, machine operating and assembling" workers (26.5\%). The subgroups of non-manual workers including "managers", "professional and related" workers, and "clerks" had a higher level of education and household income than the manual working groups in both the male and female workers. Several studies have shown an inverse association between socioeconomic status and the metabolic syndrome in women, but no association in men [16-18]. However, a recent study of the Korean population using 2007-2008 KNHANES data has reported that socioeconomic status (SES) had a positive association with the metabolic syndrome for men and an inverse association for women [19]. The researchers suggested differences in health behaviors including smoking and drinking, food consumption, heath care assessment, and psychological stress according to SES as possible explanations for the inverse association in women. The authors explained that the finding of high prevalence of metabolic syndrome in men with highest household income was consistent with an earlier study using data from the 1998 to 2001 KNHANES, which showed a positive relationship between obesity and higher SES in men [20]. This positive association among men might be applied to explain the data on the male workers in our study. The subgroups of non-manual male workers including "managers", "professional and related" workers, and "clerks" had a higher proportion of overweight and obese individuals than the other occupational groups in our study.

Among the subgroups of male manual workers, only "equipment, machine operating and assembling" workers showed a significantly higher prevalence of the metabolic syndrome than the prevalence for the male workers overall before and after age adjustment, and the prevalence was similar to the prevalence in non-manual workers. This observation was in concordance with a previous study in Spain, which showed the highest prevalence of the metabolic syndrome in the "machine installers, operators and assemblers" group among all of the occupational groups [8]. When the demographic characteristics of this group were considered, they were found to have a higher level of education (high school education and more) and household income (middle to high) in the manual worker groups. It seems that higher socioeconomic status, among other factors, might have influenced this result.

Among the female workers, although the unadjusted prevalence was greatest in the manual workers (28.4\%) and lowest in the non-manual workers (7.3\%), this difference disappeared after age standardization $(20.8 \%$ versus $20.8 \%$, respectively). Among the subgroups of the female non-manual workers, "professional and related" workers showed the lowest unadjusted prevalence of the metabolic syndrome $(7.3 \%)$ and the highest age-adjusted prevalence $(27.9 \%)$. This was the main cause for the increase in the overall prevalence in non-manual workers after age adjustment. Although not presented in this paper, the age distribution of female "professional and related" workers was skewed toward younger ages. At this age group, the prevalence of the metabolic syndrome was lower, but compared to the younger group, the prevalence in the older age group was extremely high. In the multiple logistic regression analysis, there was no significant association found between occupational groups and the metabolic syndrome in female workers.

Unlike the prevalence estimate of the male "skilled agricultural, forestry and fishery" workers, which was significantly lower than the overall male prevalence after age adjustment, female workers of the same occupational group continued to show significantly higher prevalence of the metabolic syndrome compared to the overall prevalence after age adjustment. This distinct feature was also evident in the analysis of individual 
components of the metabolic syndrome. Female "skilled agricultural, forestry and fishery" workers presented the highest age-adjusted prevalence of abdominal obesity and low HDL-cholesterol levels among the occupational subgroups. Conversely, male "skilled agricultural, forestry and fishery" workers showed the lowest age-adjusted prevalence of low HDL-cholesterol levels and a relatively lower prevalence of abdominal obesity. These gender differences may come from post-menopausal hormonal changes in female workers, given that recent studies have shown that the prevalence of the metabolic syndrome was higher in postmenopausal women than in premenopausal women [21-24]. In the present study, "skilled agricultural, forestry and fishery" workers were the most aged group; that is, more female workers of that group could be expected to be post-menopausal based on their age than in the other groups.

We categorized service and sales workers into a separate group, apart from the non-manual and manual workers. Our results showed that service/sales workers had characteristics intermediate to the other two occupational groups with regard to SES. Likewise, the ageadjusted prevalence of the metabolic syndrome among the male service/sales workers was between the prevalence in non-manual workers and the prevalence in manual workers. In the women also, considering that the dramatic increase of the prevalence in non-manual workers after age adjustment was mainly due to the uneven distribution of age, the prevalence of the metabolic syndrome in service/sales workers stood between those of manual and non-manual workers.

In this study, the work schedule pattern was included as a covariate. In both male and female workers, the percentages of night/overnight or shift work were the highest for the service workers. Generally, it is known that shift work causes the disturbance of sleep and normal circadian rhythms, and it may increase psychosocial stress, predisposing the worker to physiological disturbances related to the metabolic syndrome and cardiovascular disease $[25,26]$. Recent studies have shown that shift work is closely related to the increased risk of the metabolic syndrome [27-31]. However, the prevalence of the metabolic syndrome in this group was not higher compared to the other occupational groups. In addition, the result of multiple logistic regression analysis did not show a significant association between the metabolic syndrome and work schedule (data not shown).

The strengths of our investigation are the use of a large sample representative of the Korean population and the analysis of the prevalence of the metabolic syndrome in 9 occupational groups considering multiple variables such as age, gender, level of education, household income, and smoking status. The limitations are an inability to draw causal inferences due to the cross- sectional design and unavailability of detailed information about work-related condition in the KNHANES.

In conclusion, our study demonstrated variability in the prevalence of the metabolic syndrome by occupational group among Korean workers. In the male workers, nonmanual workers appeared to be more vulnerable to the metabolic syndrome compared to service/sales workers or manual workers, but not in the female workers. Future research should evaluate factors that may influence the occurrence of the metabolic syndrome in each occupational group so that our findings can be utilized to establish appropriate preventive measures for the metabolic syndrome and co-occurring diseases including cardiovascular disease and diabetes.

\section{Competing interests}

The authors declare that they have no competing interests.

\section{Authors' contributions}

$\mathrm{DH}$ Kim and JY Ryu designed the research. S Hong and $\mathrm{CH}$ Kim collected the data. JY Ryu and S Lee performed the statistical analysis. JY Ryu, JT Lee, JH Kim, and DH Kim interpreted the data. JY Ryu and S Lee wrote the manuscript. All of the authors read and approved the final manuscript.

\section{Author details}

${ }^{1}$ Department of Occupational and Environmental Medicine, Inje University Haeundae Paik Hospital, 875 Heaundae-ro, Haeundae-Gu, Busan 612-862, South Korea. ${ }^{2}$ Inje University Graduate School, 197 Inje-ro, Gimhae, Gyungnam, South Korea. ${ }^{3}$ Department of Occupational and Environmental Medicine, College of Medicine \& Institute of Environmental and Occupational Medicine, Inje University, 75 Bokji-roBusanjin-gu, Busan, South Korea.

Received: 5 April 2013 Accepted: 1 July 2013

Published: 5 August 2013

\section{References}

1. Kassi E, Pervanidou P, Kaltsas G, Chrousos G: Metabolic syndrome: definitions and controversies. BMC Med 2011, 9:48.

2. Isomaa B, Almgren P, Tuomi T, Forsen B, Lahti K, Nissen M, Taskinen MR, Groop L: Cardiovascular morbidity and mortality associated with the metabolic syndrome. Diabetes Care 2001, 24:683-689.

3. Haffner SM, Valdez RA, Hazuda HP, Mitchell BD, Morales PA, Stern MP: Prospective analysis of the insulin-resistance syndrome (syndrome $\mathrm{X}$ ). Diabetes 1992, 41:715-722.

4. Neira CP, Hartig M, Cowan PA, Velasquez-Mieyer PA: The prevalence of impaired glucose metabolism in Hispanics with two or more risk factors for metabolic syndrome in the primary care setting. J Am Acad Nurse Pract 2009, 21:173-178.

5. Park HS, Kim SM, Lee JS, Lee J, Han JH, Yoon DK, Baik SH, Choi DS, Choi KM: Prevalence and trends of metabolic syndrome in Korea: Korean National Health and Nutrition Survey 1998-2001. Diabetes Obes Metab 2007, 9:50-58.

6. Lim S, Shin H, Song JH, Kwak SH, Kang SM, Won Yoon J, Choi SH, Cho SI, Park KS, Lee HK, et al: Increasing prevalence of metabolic syndrome in Korea: the Korean National Health and Nutrition Examination Survey for 1998-2007. Diabetes Care 2011, 34:1323-1328.

7. Park HS, Park CY, Oh SW, Yoo HJ: Prevalence of obesity and metabolic syndrome in Korean adults. Obes Rev 2008, 9:104-107.

8. Sanchez-Chaparro MA, Calvo-Bonacho E, Gonzalez-Quintela A, FernandezLabandera C, Cabrera M, Sainz JC, Fernandez-Meseguer A, Banegas JR, Ruilope LM, Valdivielso P, Roman-Garcia J: Occupation-related differences in the prevalence of metabolic syndrome. Diabetes Care 2008, 31:1884-1885

9. Davila EP, Florez H, Fleming LE, Lee DJ, Goodman E, LeBlanc WG, Caban-Martinez AJ, Arheart KL, McCollister KE, Christ SL, et al: Prevalence of the metabolic syndrome among U.S. workers. Diabetes Care 2010, 33:2390-2395. 
10. Myong JP, Kim HR, Jung-Choi K, Baker D, Choi B: Disparities of metabolic syndrome prevalence by age, gender and occupation among Korean adult workers. Ind Health 2012, 50:115-122.

11. KOREAN STANDARD CLASSIFICATION OF OCCUPATIONS. http://kostat.go.kr/ e_book/kssc/KSCO07/EBook.htm.

12. Alberti KG, Zimmet $P$, Shaw J: The metabolic syndrome-a new worldwide definition. Lancet 2005, 366:1059-1062.

13. Grundy SM, Brewer HB Jr, Cleeman Jl, Smith SC Jr, Lenfant C: Definition of metabolic syndrome: Report of the National Heart, Lung, and Blood Institute/American Heart Association conference on scientific issues related to definition. Circulation 2004, 109:433-438.

14. Rosner BA: Fundamentals of Biostatistics. 6th edition. Pacific Grove, CA, Brooks: Duxbury Press; 2005.

15. Choi B, Schnall PL, Yang H, Dobson M, Landsbergis P, Israel L, Karasek R, Baker D: Sedentary work, low physical job demand, and obesity in US workers. Am J Ind Med 2010, 53:1088-1101.

16. Loucks EB, Magnusson KT, Cook S, Rehkopf DH, Ford ES, Berkman LF: Socioeconomic position and the metabolic syndrome in early, middle, and late life: evidence from NHANES 1999-2002. Ann Epidemio/ 2007, 17:782-790.

17. Zhan Y, Yu J, Chen R, Gao J, Ding R, Fu Y, Zhang L, Hu D: Socioeconomic status and metabolic syndrome in the general population of China: a cross-sectional study. BMC Publ Health 2012, 12:921.

18. Dallongeville J, Cottel D, Ferrieres J, Arveiler D, Bingham A, Ruidavets JB, Haas B, Ducimetiere $P$, Amouyel P: Household income is associated with the risk of metabolic syndrome in a sex-specific manner. Diabetes Care 2005, 28:409-415.

19. Park SJ, Kang HT, Nam CM, Park BJ, Linton JA, Lee YJ: Sex differences in the relationship between socioeconomic status and metabolic syndrome: the Korean National Health and Nutrition Examination Survey. Diabetes Res Clin Pract 2012, 96:400-406.

20. Yoon YS, Oh SW, Park HS: Socioeconomic status in relation to obesity and abdominal obesity in Korean adults: a focus on sex differences. Obesity (Silver Spring) 2006, 14:909-919.

21. Jouyandeh Z, Nayebzadeh F, Qorbani M, Asadi M: Metabolic syndrome and menopause. J Diabetes Metab Disord 2013, 12:1.

22. Zivkovic TB, Vuksanovic M, Jelic MA, Stojanovic J, Buric B, Jojic B, Milic N, Vujovic S: Obesity and metabolic syndrome during the menopause transition in Serbian women. Climacteric 2011, 14:643-648

23. Figueiredo Neto JA, Figueredo ED, Barbosa JB, Barbosa Fde F, Costa GR, Nina VJ, Nina RV: Metabolic syndrome and menopause: cross-sectional study in gynecology clinic. Ara Bras Cardiol 2010, 95:339-345.

24. Lobo RA: Metabolic syndrome after menopause and the role of hormones. Maturitas 2008, 60:10-18.

25. Puttonen S, Harma M, Hublin C: Shift work and cardiovascular disease pathways from circadian stress to morbidity. Scand I Work Environ Health 2010, 36:96-108

26. Puttonen S, Viitasalo K, Harma M: The relationship between current and former shift work and the metabolic syndrome. Scand J Work Environ Health 2012, 38:343-348.

27. Esquirol Y, Bongard V, Mabile L, Jonnier B, Soulat JM, Perret B: Shift work and metabolic syndrome: respective impacts of job strain, physical activity, and dietary rhythms. Chronobiol Int 2009, 26:544-559.

28. Sookoian S, Gemma C, Pirola CJ, Gonzalez CD, Alvarez A, Burgueno A, Fernandez Gianotti T: Effects of rotating shift work on biomarkers of metabolic syndrome and inflammation. J Intern Med 2007, 261:285-292.

29. De Bacquer D, Van Risseghem M, Clays E, Kittel F, De Backer G, Braeckman $L$ : Rotating shift work and the metabolic syndrome: a prospective study. Int J Epidemiol 2009, 38:848-854.

30. Pietroiusti A, Neri A, Somma G, Coppeta L, lavicoli I, Bergamaschi A, Magrini A: Incidence of metabolic syndrome among night-shift healthcare workers. Occup Environ Med 2010, 67:54-57.

31. Lin YC, Hsiao TJ, Chen PC: Persistent rotating shift-work exposure accelerates development of metabolic syndrome among middle-aged female employees: a five-year follow-up. Chronobiol Int 2009, 26:740-755

doi:10.1186/2052-4374-25-13

Cite this article as: Ryu et al:: Prevalence of the Metabolic Syndrome among Korean Workers by Occupational Group: Fifth Korean National Health and Nutrition Examination Survey (KNHANES) 2010. Annals of Occupational and Environmental Medicine 2013 25:13.

\section{Submit your next manuscript to BioMed Central and take full advantage of:}

- Convenient online submission

- Thorough peer review

- No space constraints or color figure charges

- Immediate publication on acceptance

- Inclusion in PubMed, CAS, Scopus and Google Scholar

- Research which is freely available for redistribution 